\title{
Stress proteins as predictors of COVID-19 outcomes
}

\author{
Barry G. Hall ${ }^{1}$
}

Accepted: 7 December 2020 / Published online: 5 January 2021

(C) Cell Stress Society International 2021

As of December 1, 2020, 14,108,490 cases of COVID-19, resulting from SARS-CoV-2 virus infections, have been reported in the USA (https://www.worldometers.info/ coronavirus/country/us/). Of the 8,609,994 closed cases, $8,333,018(97 \%)$ of the patients have recovered and 276,976 (3\%) patients have died. COVID-19 disease is remarkable for the range of responses to infection, ranging from asymptomatic, through severe respiratory disease, through death. Initial findings suggested that severe infections were limited to the elderly and to those with preexisting conditions such as heart disease, hypertension, diabetes, or obesity, but more recently it has been realized that severe infections, some leading to death, occur in those in their 20s and 30 s, and even in young children.

Severe infections appear to involve a hyper-autoimmune response, including a "cytokine storm" leading to multiple organ failure (De Maio and Hightower 2020). That led Cappello (2020) to ask if "the virus employs molecular mimicry to trick the patient's immune systems to produce antibodies against viral protein epitopes capable of cross-reacting to self-proteins" (Hightower and Santoro 2020). Cappello found 17 molecular chaperones that shared peptides found among SARS-CoV-2 proteins. Zimmermann et al. (2020) found that the level of HSP27 is a predictor of acute COPD exacerbation, a severe pulmonary disease that shares many characteristics with severe COVID-19 disease.

The above observations suggest a possible association between the productions of one or more stress proteins and severity of COVID-19 disease. That in turn suggests the possibility of a quantitative relationship between cellular or extracellular stress proteins that could be used to predict the severity of COVID-19 cases.

I suggest that an immediate study is warranted to determine the relationship between stress proteins and the outcomes of

Barry G. Hall

barryghall@gmail.com

1 Bellingham Research Institute, Portland, OR, USA
COVID-19 cases. The current surge of COVID-19 cases provides an opportunity to determine that relationship fairly easily. I suggest that a large number of subjects currently being tested for COVID-19 could be asked to volunteer to provide a small blood sample from which the amount of several stress proteins could be determined. A couple of hundred volunteers who test negative would serve as a control to determine the normal baseline for each stress protein, while 300 volunteers who test positive would serve to determine which stress proteins effectively predict COVID-19 outcomes. Those who test positive but are asymptomatic and do not develop symptoms later serve to determine a level that predicts harmless infections. Those should be followed up with another such blood test 2 or 3 weeks later. Those who develop symptoms within 14 days should be tested again for stress proteins upon developing symptoms and tested again when the symptoms appear to have disappeared. Those whose symptoms are severe enough to warrant hospitalization should be tested daily until discharged or death occurs.

In such an initial study, the stress protein levels should be evaluated by gel electrophoresis because we do not know at this time which stress protein levels are best associated with COVID-19 outcomes. Gel electrophoresis is not terribly quantitative, but it should be sufficient to identify the best candidates for stress proteins that predict COVID-19 outcomes. Once a few candidate stress proteins are identified, ELISA assays should be retrospectively applied to the stored blood samples using those candidates to firmly establish the predictive value of the candidate stress proteins.

Determining the relationships of stress proteins to COVID19 outcomes is certainly of scientific interest in understanding COVID-19 disease, but that understanding is of practical value only if those predicted outcomes can be used to intervene in the disease progress. DeMaio and Hightower (2020) have pointed out that SARS-Cov-2-mediated pneumonia often results in dramatic reductions in arterial oxygen levels, sometimes to levels of $50 \%$, resulting in an uncontrolled inflammatory response that ends in shock, multiple organ failure, and death. The response, termed acute respiratory distress syndrome, or ARDS, is not unique to COVID-19 disease but also 
occurs in other infections. Early intervention is thought to be critical to ameliorating the condition (Cauvi et al. 2012). Hyperbaric oxygen therapy (HBOT) has been shown to dramatically improve the outcome of ARDS resulting from sepsis (reviewed in DeMaio and Hightower 2020). Early intervention with HBOT in COVID-19 cases predicted to have serious outcomes could save lives. Indeed, as that and other therapies are discovered, the ability to predict likely outcomes based on the levels of predictive stress proteins could ensure that therapeutic resources are deployed efficiently and effectively.

The study outlined above should be initiated immediately on a scale that will provide confidence in the results. Such a study, involving only 500-1000 patients, could be accomplished within a period of 8 weeks given sufficient resources. Is it reasonable to ask the government to support such a study when we do not yet know if stress proteins will actually predict COVID-19 outcomes? Yes! The USA has committed billions to manufacturing and preparing to distribute vaccines before they have been determined to be effective and safe. Now that we are on the brink of approving at least three such vaccines, the willingness to commit vast funds prior to certainty about effectiveness will save months in vaccinating the public and will save hundreds of thousands of lives. I estimate that funding at the level of less than two million dollars would be required to carry the proposed study. This approach could likewise save lives by permitting early therapeutic interventions and optimizing the use of therapeutic resources.

\section{References}

Cappello F (2020) IS COVID-19 a proteiform disease inducing also molecular mimicry phenomena? Cell Stress Chaperones 25(4):381382

Cauvi DM, Song D, Vazquez DE, Hawisher D, Bermudez JA, Williams MR, Bickler S, Coimbra R, deMaio A (2012) Period of irreversible therapeutic intervention during sepsis correlates with phase of innate immune dysfunction. J Biol Chem 287:19804-19815. https://doi. org/10.1074/jbc.M112.359562

De Maio A, Hightower LE (2020) COVID-19, acute respiratory distress syndrome (ARDS), and hyperbaric oxygen therapy (HBOT): what is the link? Cell Stress Chaperones 25(5):717-720

Hightower LE, Santoro MG (2020) Coronaviruses and stress: from cellular to global. Cell Stress Chaperones 25(5):701-705

Zimmermann M, Traxler D, Bekos C, Simader E, Mueller T, Graf A, Lainscak M, Marcun R, Kosnik M, Felzar M et al (2020) Heat shock protein 27 as a predictor of prognosis in patients admitted to hospital with acute COPD exacerbation. Cell Stress Chaperones 25(1):141149

Publisher's note Springer Nature remains neutral with regard to jurisdictional claims in published maps and institutional affiliations. 